\title{
Characteristics Relating to Adherence and Persistence to Basal Insulin Regimens Among Elderly Insulin- Naïve Patients with Type 2 Diabetes: Pre-Filled Pens versus Vials/Syringes
}

\author{
S. Lane Slabaugh · Jonathan R. Bouchard • Yong Li • \\ Jean C. Baltz · Yunus A. Meah · D. Chad Moretz \\ To view enhanced content go to www.advancesintherapy.com \\ Received: September 24, 2015 / Published online: November 13, 2015 \\ (C) The Author(s) 2015. This article is published with open access at Springerlink.com
}

\begin{abstract}
Introduction: Previous studies have found higher rates of adherence in patients with type 2 diabetes mellitus (T2DM) using insulin pens compared to vial and syringe administration; however, little evidence is available to support this observation in elderly patients.
\end{abstract}

Methods: This was a retrospective claims database analysis of a predominantly elderly Medicare Advantage with Prescription Drug (MAPD) insurance population consisting of 3172 insulin-naïve patients with T2DM who initiated basal insulin using pre-filled pens or

Electronic supplementary material The online version of this article (doi:10.1007/s12325-015-0266-5) contains supplementary material, which is available to authorized users.

S. L. Slabaugh $(\bowtie) \cdot$ Y. A. Meah

Humana, Inc., Louisville, KY, USA

e-mail: sslabaugh@humana.com

J. R. Bouchard

Novo Nordisk Pharmaceuticals, Inc., Plainsboro, NJ, USA

Y. Li · D. C. Moretz

Comprehensive Health Insights, Inc., Louisville, KY, USA

J. C. Baltz

Mountain Diabetes, Asheville, NC, USA vial and syringe ('vial'). The index date was defined by the first pharmacy claim for basal insulin. Adherence, measured as proportion of days covered (PDC) and medication possession ratio (MPR), and persistence were evaluated in a 12-month follow-up period using an adjusted days' supply. Multivariate regression analyses and a Cox proportional hazards model were used to identify characteristics associated with adherence and non-persistence, respectively, and compare findings between the pen and vial groups.

Results: The pen cohort was slightly younger than the vial cohort (69.4 vs. 70.1 years, respectively; $P=0.0338)$. Similar proportions of male patients $(53.3 \%$ vs. $56.8 \% ; P=0.0529)$ occurred in both cohorts, and lower Deyo-Charlson Comorbidity Index (4.4 vs. 5.0; $P<0.0001)$ was found for the pen cohort. Adjusted mean PDC was significantly higher in the pen cohort than the vial cohort (0.67 vs. $0.50 ; P<0.001)$, as was mean MPR $(0.75$ vs. $0.57 ; P<0.0001)$. Adjusted odds for adherence (PDC $\geq 80 \%$ ) showed a positive association with use of an insulin pen (odds ratio $=2.19,95 \% \mathrm{CI}$ : 1.86-2.59). The adjusted risk of non-persistence (discontinuation) was significantly lower (58\%) 
in the pen cohort relative to the vial cohort (hazard ratio $=0.42$, 95\% CI: 0.38-0.45). Key limitations include assumptions related to accuracy and comprehensiveness of claims data, and specifically days' supply data used to measure insulin adherence.

Conclusion: These findings suggest that pen devices improved insulin therapy adherence in a primarily elderly MAPD population with T2DM.

Funding: Novo Nordisk Pharmaceuticals, Inc.

Keywords: Adherence; Basal insulin; Elderly; Insulin detemir; Insulin glargine; Insulin isophane; Insulin-naïve; Pen; Type 2 diabetes mellitus

\section{INTRODUCTION}

The incidence of complications in patients with type 2 diabetes mellitus (T2DM) can be reduced by appropriate glycemic control, and there is general consensus that insulin therapy should be started in patients who fail to achieve glycemic control targets with one or more non-insulin antidiabetic agents [1-3]. Lower comorbidity prevalence and severity have been reported for patients with early adoption and high adherence to insulin therapy [4]. However, patients and providers often delay insulin initiation due to well-described barriers, such as fear of needles or the inconvenience of injections compared to oral therapies [5]. Insulin therapy is also associated with potentially serious hypoglycemic consequences if not managed properly. Pre-filled, disposable insulin pens, which have been available since the 1980s, offer an administration option that may be considered preferable by some patients. Previous reports have shown improved adherence and persistence primarily in commercially insured patients using insulin pen devices compared to those using traditional vial and syringe administration [6-8].

In elderly or debilitated patients, in particular, pre-filled insulin pens may offer advantages such as improved dose accuracy and consistency, greater convenience, greater ease of physical manipulation, and use needles that incur less pain upon injection [9]. However, pen devices are typically more costly, may take longer to administer, and are not available for all insulins [9]. Ultimately, the consistency with which patients use an administration device will affect the clinical results achieved. Prior studies evaluating adherence and persistence with insulin pens versus vials have typically evaluated single insulin products and have been based on general diabetes populations, not specifically the elderly $[6,7,10-12]$. One exception is a recent study by Miao et al. [13] that reported improved persistence and adherence to insulin administration in elderly Medicare patients with T2DM who initiated or switched to disposable pens compared with vial and syringe. Even less is known about the drivers of adherence to insulin therapy in elderly patients with T2DM who are likely to have higher mortality and complications compared with younger patients with T2DM and age group peers without T2DM [14-16].

The objective of this study was to evaluate patient characteristics associated with adherence and persistence to insulin therapy in a Medicare Advantage with Prescription Drug (MAPD) insurance population comprised primarily of elderly health plan members who were insulin-naïve and who started a basal insulin regimen using disposable pen or vial and syringe. 


\section{METHODS}

\section{Data Source}

This was a retrospective analysis using medical and pharmacy claims, laboratory results, and patient characteristics obtained from a large US national health plan (Humana, Inc.). The study was limited to claims of Humana members enrolled in an MAPD plan. The research protocol was reviewed and approved prior to study initiation by an independent Institutional Review Board and was granted a waiver of Health Insurance Portability and Accountability Act authorization and a waiver of informed consent.

\section{Subject Selection}

The criteria for patient selection included: age between 18 and 89 years; evidence of basal insulin use [insulin detemir, insulin glargine, or insulin isophane (NPH)]; and a diagnosis of T2DM at any time during the identification period (July 1, 2010 through September 30, 2011). T2DM was defined by any of the following criteria: (1) two or more prescription fills for oral antidiabetic medications (OADs) or a glucagon-like peptide-1 agonist; (2) one or more medical claims with a primary diagnosis of T2DM [International Classification of Diseases, Ninth Revision, Clinical Modification (ICD-9-CM) code 250.x0 or 250.x2]; or (3) two or more medical claims with a secondary diagnosis of T2DM on different dates. The index date for each patient was defined as the first pharmacy claim for basal insulin during the identification period. All patients were required to have at least 12 months of continuous enrollment for the 12 months before and after the index date. The attrition diagram (Fig. 1) outlines additional inclusion and exclusion criteria.

\section{Baseline Characteristics}

Baseline demographic characteristics examined included age, sex, geographic region (Northeast, Southeast, Midwest, and West), population density (urban, suburban, and rural), race/ ethnicity (White, Black, Hispanic, and other), and plan characteristics [low income subsidy (LIS) status and dual eligibility for Medicare and Medicaid]. Population density was assigned by matching patient zip codes to Rural-Urban Commuting Area (RUCA) codes [17] and applying the Washington State Department of Health's RUCA consolidation system [18].

Clinical characteristics measured during the baseline period included the Deyo-Charlson Comorbidity Index (Deyo-CCI) [19]. The CCI is a disease-based indicator derived from data from medical records, which was designed to assess the risk of 1-year mortality among inpatients on a medical service. The Deyo-CCI is an adaptation of the CCI, which adjusts the index to administrative claims research via mapping to ICD-9-CM diagnoses and procedure codes. Clinical characteristics were also measured using an adapted diabetes complications severity index (DCSI), which quantifies the severity of a wide range of diabetes complications. The adapted version has been proven to be accurate using claims data in the absence of laboratory data [20,21]. Further characteristics examined included use of non-insulin injectable products during the pre-index period, oral antidiabetic use during the pre-index period, prescribing physician specialty, pre-index count of glycated hemoglobin (HbA1c) tests performed, number of physician office visits, and number of prescription claims. 


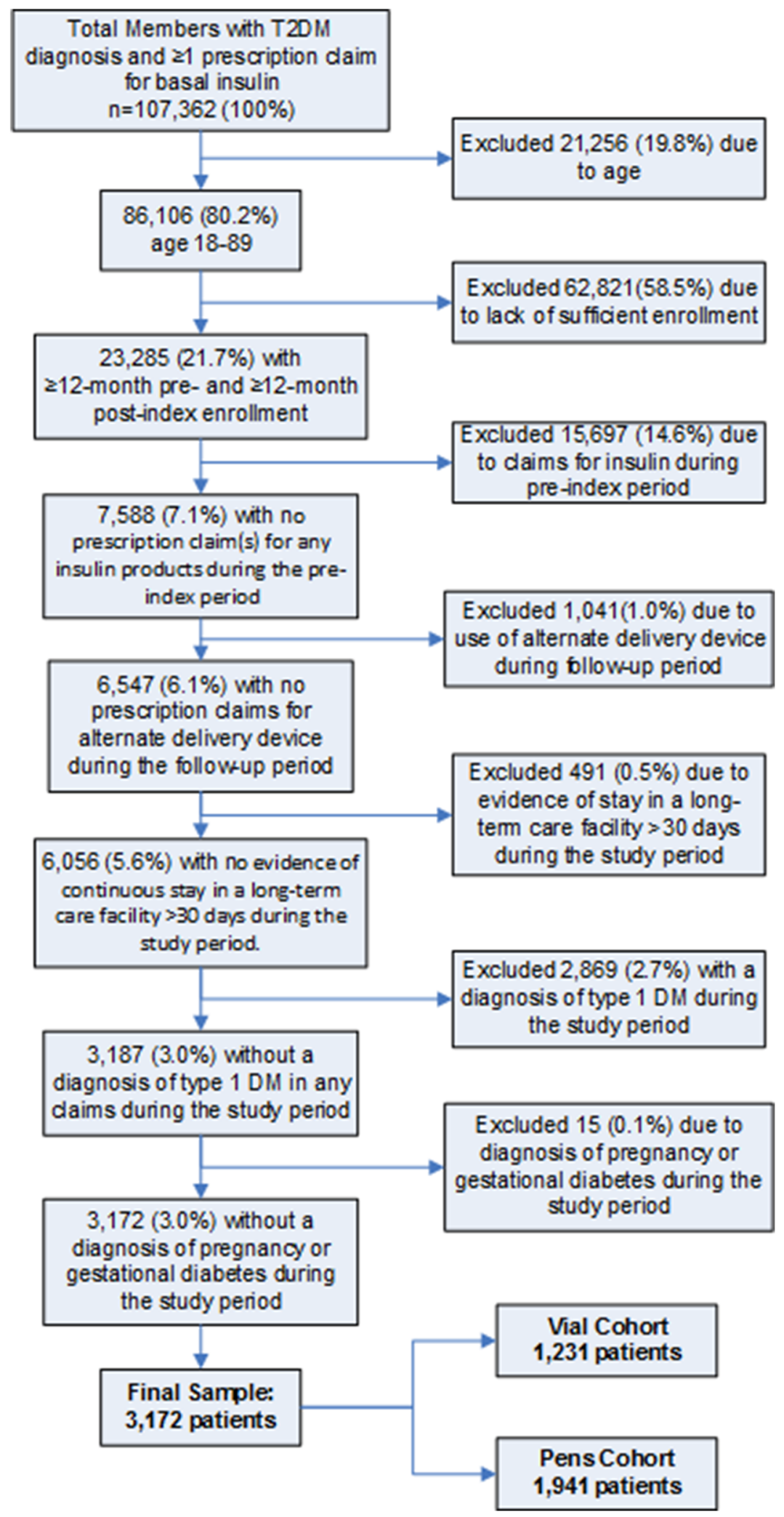

Fig. 1 Attrition diagram. T2DM type 2 diabetes mellitus 


\section{Outcome Measures}

Outcomes of interest included adherence, persistence, and time-to-discontinuation. Due to the customization of insulin dosing, differences in package sizes (commonly 1000 units for insulin vials and 1500 units in a package of insulin pens), expiration dates, patient usage, and inaccuracies in the days' supply entered at pharmacies, it can be problematic to use the days' supply field directly from pharmacy claims when calculating adherence and persistence to insulin. Despite these differences, insulin prescriptions are most often dispensed as a 30 days' supply. This presents a challenge when making comparisons of adherence and persistence between basal insulin supplied as insulin-filled pen devices and that supplied in vials. To address these issues, a days' supply adjustment factor was calculated for each basal insulin-delivery device combination (6 in total) in a manner similar to that described by Buysman et al. [6]. A unique adjustment factor was established for each insulin-delivery device combination because each insulin may be packed in quantities different from the others, have a different shelf-life, and/or be dosed differently by providers. Specifically, the following steps were followed to calculate the adjusted days' supply, as a measure of adherence for all basal insulin pharmacy claims:

1. Patients from the study sample with $\geq 2$ prescription claims for basal insulin were identified. Patients in this sample were required to be insulin-naïve prior to the index date and meet all other study criteria described above in subject selection.

2. Days' supply reported on each pharmacy claim and the time between pharmacy claims for basal insulin was determined for these patients.
3. The adjustment factor was calculated as the ratio of the median time between insulin claims divided by the median pharmacy reported days' supply for each basal insulin-delivery device combination.

4. An adjusted days' supply was calculated for the final study sample by multiplying the actual days' supply reported on each pharmacy claim by the adjustment factor for the appropriate basal insulin-delivery device combination. The adjustment factors were 1.80 and 1.30 for pen and vial, respectively, for insulin glargine; 1.87 and 1.33 , respectively, for insulin detemir; and 1.73 and 1.37, respectively, for insulin isophane (NPH).

Adherence was measured using the proportion of days covered (PDC), as well as the medication possession ratio (MPR). The PDC is a newer and more conservative measure of adherence than the MPR $[22,23]$. The PDC and MPR are similar in that the days' supply from pharmacy claims is used to calculate adherence for both, but they differ in their approach to determining how days' supply is used to calculate the numerator used for the calculations. MPR is most commonly calculated by summing the days' supply from all claims for a study drug and then dividing by the number of days from drug initiation to the end of drug persistence. Because all days' supplies are included, even when early refills occur or a patient switches medications within the same class, the ratio of days' supply to days in the study period can potentially be $>1.0$. When calculating PDC, the numerator is the total number of covered days during the study period, regardless of how many prescription claims are available on any single day. Each day in the study period is independently evaluated for coverage by the study drug, and the maximum PDC is therefore 1.0 . 
The covered days for each prescription began on the fill date and lasted for the duration of the adjusted days' supply. Days spent in a hospital setting were counted as days with medication available. Days of insulin possession from a previous fill that overlapped with a subsequent fill were not double-counted, but they were instead removed from the calculation of the adherence measures. Furthermore, no adjustment to the start date of a new prescription was made in instances where there were overlapping days for the same medication. Adherence was reported as both a continuous variable (0-100\%) and a dichotomous one with a threshold of $\geq 80 \%$ PDC considered 'adherent.' Persistence was measured as the number of days of continuous basal insulin coverage (using adjusted days' supply) prior to a gap in medication coverage. Given the use of adjustment factors in the calculation in the adjusted days' supply, a conservative gap threshold of one or more days was employed to identify non-persistence [6].

Examinations of adherence and persistence to insulin that use the reported days' supply directly from pharmacy claims data without adjusting for the weaknesses mentioned are of limited value. Their weaknesses include varying package sizes and dosing schedules, which are frequently not accounted for in the reported days' supply. Pharmacy claims most frequently report a 30 days' supply even if the patient will have insulin left after 30 days if taken at the prescribed dose. For this reason, the present study used adjustment factors to accommodate for the difficulties in calculating adherence to insulin using a claims database. A unique adjustment factor was calculated for each of the 6 basal insulin-delivery device combinations included in this study, and all adjustment factors were $>1$, thereby lengthening the days' supply for all of the basal insulin pharmacy claims.

The change in the HbA1c value from the pre-index period to the post-index period was not a primary outcome measure, and therefore not all patients in the study were required to have both pre-index and post-index HbA1c values. However, among patients with laboratory values available in both time periods, this measure was assessed as a measure of glycemic control.

\section{Statistical Analysis}

Univariate descriptive statistics were used to compare demographic (age, sex, race, etc.) and clinical characteristics (Deyo-CCI, oral antidiabetic use during the baseline period, number of physician office visits, etc.) between the two study cohorts. Differences in baseline characteristics were assessed using ANOVA for continuous variables (age and comorbidity index score) and Chi-square tests for categorical variables (sex and geographic region). The difference in HbA1c from baseline to follow-up was assessed using Student's $t$ test.

The study compared mean PDC (as a measure of adherence) and mean persistence (in days) between the two study cohorts (pen vs. vial) using $t$ tests. The proportion of each study cohort above the adherence threshold of $80 \%$ was compared using the Chi-square test. A cutoff of $80 \%$ is frequently used as the threshold when measuring adherence as a dichotomous variable [6]. MPR values were also calculated and compared between the two cohorts in a similar fashion.

Multivariable regression analyses were performed to identify characteristics associated with increased adherence using linear regression for continuous PDC and logistic regression for dichotomous adherence with an 
80\% PDC threshold. Persistence was analyzed using a Cox proportional hazards model. All reported demographic and clinical characteristics, including whether the patient was classified in the pen or vial cohort, were added to the statistical models. All analyses of data were conducted using SAS version 9.2 (SAS Institute Inc., Cary, NC, USA). The a priori alpha level for all inferential analyses was set at 0.05, with all statistical tests being two-tailed, unless otherwise specified.

\section{RESULTS}

A sample of 3172 MAPD patients meeting all study criteria was identified, including 1941 in the pen cohort, and 1231 in the vial cohort. The demographic and clinical characteristics of the two cohorts are presented in Tables 1 and 2 . Mean age in the pen cohort was 69.4 years compared with 70.1 years in the vial cohort $(P=0.0338)$, and the percentage of males was similar in the two groups $(53.3 \%$ and $56.8 \%$, respectively; $P=0.0529$ ). A significantly higher proportion of the pen cohort patients resided in the Midwest compared to the vial cohort (29.4\% vs. $18.2 \% ; P<0.0001)$ while a lower proportion resided in the South $(59.3 \%$ vs. 71.3\%; $\quad P<0.0001)$. A significantly higher proportion of the pen cohort was made up of patients classified as White $(80.8 \%$ vs. $75.4 \%)$, while a lower proportion was comprised of patients classified as Black (14.3\% vs. $18.3 \%)$ and Hispanic ( $1.8 \%$ vs. $3.2 \% ; P=0.0009$ for all). Pen use was more prevalent in suburban areas (25.6\% vs. $21.4 \%$ ) and less prevalent in urban areas $(63.7 \%$ vs. $67.0 \% ; P=0.0037$ for all). Equal proportions of the rural areas used both delivery devices.

Both the Deyo-CCI and DSCI were significantly lower in the pen cohort compared with the vial cohort (Deyo-CCI: 4.4 vs. $5.0 ; P<0.0001$; DCSI: 3.8 vs. $4.3 ; P<0.0001$; Table 2). A large majority of patients was prescribed insulin by a primary care physician (PCP; pen 76.4\%, vial 78.1\%). However, a higher proportion of the pen cohort compared with the vial cohort was prescribed index basal insulin by an endocrinologist (7.3\% vs. $3.7 \%$; $P<0.0001)$, and a smaller proportion received prescriptions from an emergency medicine practitioner $(0.5 \%$ vs. $1.5 \% ; P=0.003)$. Use of oral antidiabetic agents during the baseline period, number of $\mathrm{HbA1c}$ tests performed, and the number of physician office visits during the baseline period were significantly higher in the pen cohort than in the vial cohort $(P<0.0001$ in all cases). All differences in baseline characteristics between the cohorts were accounted for in the multivariate models used to compare adherence and persistence as measured by the PDC.

\section{Adherence}

Results of the adherence analyses are presented in Table 3. Unadjusted and adjusted PDC values were significantly higher in the pen cohort than in the vial cohort (unadjusted PDC, $71 \%$ vs. 53\%; $P<0.001$; adjusted PDC, $67 \%$ vs. $50 \%$; $P<0.001)$, as were the MPR values $(0.75$ vs. $0.57 ; P<0.0001)$. The proportion of patients with a PDC $\geq 80 \%$ was significantly higher in the pen versus the vial cohort $(49.4 \%$ vs. $29.7 \%$; $P<0.001)$, as was the proportion of patients with an MPR $\geq 80 \% \quad(57.9 \%$ vs. $35.4 \%$; $P<0.0001)$.

On multivariate analysis, the adjusted odds of being adherent (PDC $\geq 80 \%$ ) showed a positive relationship between use of an insulin pen and being adherent (odds ratio $=2.19$, 95\% CI: 1.86-2.59). No significant difference in the odds of adherence was seen between 
Table 1 Baseline demographic characteristics

\begin{tabular}{|c|c|c|c|}
\hline Characteristic & Pen cohort $(n=1941)$ & Vial cohort $(n=1231)$ & $P$ value \\
\hline Age, years, mean (SD) & $69.4(8.6)$ & $70.1(8.6)$ & 0.0338 \\
\hline Male gender, $n(\%)$ & $1034(53.3)$ & $699(56.8)$ & 0.0529 \\
\hline Geographic region, $n(\%)$ & & & $<0.0001$ \\
\hline Northeast & $37(1.9)$ & $18(1.5)$ & \\
\hline Midwest & $571(29.4)$ & $224(18.2)$ & \\
\hline South & $1151(59.3)$ & $878(71.3)$ & \\
\hline West & $182(9.4)$ & $111(9.0)$ & \\
\hline Population density, $n$ (\%) & & & 0.0037 \\
\hline Urban & $1236(63.7)$ & $825(67.0)$ & \\
\hline Suburban & $497(25.6)$ & $264(21.4)$ & \\
\hline Rural & $200(10.3)$ & $127(10.3)$ & \\
\hline Race/ethnicity, $n$ (\%) & & & 0.0009 \\
\hline White & $1568(80.8)$ & $928(75.4)$ & \\
\hline Black & $277(14.3)$ & $225(18.3)$ & \\
\hline Hispanic & $35(1.8)$ & $39(3.2)$ & \\
\hline Other & $61(3.1)$ & $39(3.2)$ & \\
\hline LIS status only, $n$ (\%) & $153(7.9)$ & $95(7.7)$ & 0.8659 \\
\hline Dual eligibility only ${ }^{\mathrm{a}}, n(\%)$ & $9(0.5)$ & $6(0.5)$ & 0.9244 \\
\hline LIS status and dual eligibility ${ }^{\mathrm{a}}, n(\%)$ & $128(6.6)$ & $107(8.7)$ & 0.0279 \\
\hline
\end{tabular}

LIS low income subsidy, $S D$ standard deviation

${ }^{a}$ Dual eligibility for Medicare and Medicaid

patients prescribed their index prescription by an endocrinologist relative to a PCP. Additional relationships are described in Table 4.

\section{Persistence}

The unadjusted mean persistence was significantly longer in the pen cohort than in the vial cohort (181.0 days vs. 93.7 days; $P<0.0001$ ), and the adjusted risk [hazard ratio (HR)] of non-persistence (discontinuation) was $58 \%$ lower $(\mathrm{HR}=0.415$, 95\% CI: 0.381-0.452; Table 3 ) in the pen cohort relative to the vial cohort. The pen cohort also had significantly more basal insulin prescription fills prior to discontinuation (mean number of claims: 2.93 vs. $2.13 ; \quad P<0.0001 ; \quad$ Table 3$)$. As with adherence, no significant difference was found in the risk of non-persistence between those prescribed their index basal insulin prescription by an endocrinologist compared to a PCP.

The Cox proportional hazard model examining the characteristics associated with non-persistence revealed that, as with adherence, no significant relationship was found between the prescribing of basal insulin 
Table 2 Baseline clinical characteristics

\begin{tabular}{|c|c|c|c|}
\hline Characteristic & Pen cohort $(n=1941)$ & Vial cohort $(n=1231)$ & $P$ value \\
\hline Deyo-CCI, mean (SD) & $4.4(2.8)$ & $5.0(3.0)$ & $<0.0001$ \\
\hline DCSI Score, mean (SD) & $3.8(2.5)$ & $4.3(2.6)$ & $<0.0001$ \\
\hline Non-insulin injectable use ${ }^{\mathrm{a}}, n(\%)$ & $226(11.6)$ & $119(9.7)$ & 0.0814 \\
\hline Oral antidiabetic use ${ }^{\mathrm{a}}, n(\%)$ & $1806(93.0)$ & $1034(84.0)$ & $<0.0001$ \\
\hline Count of $\mathrm{HbAlc}$ tests performed ${ }^{\mathrm{a}}$, mean $(\mathrm{SD})$ & $3.7(2.3)$ & $3.3(2.7)$ & $<0.0001$ \\
\hline Number of physician office visits ${ }^{\mathrm{a}}$, mean (SD) & $35.7(29.0)$ & $33.4(31.3)$ & 0.0001 \\
\hline Number of prescription claims ${ }^{\mathrm{a}}$, mean (SD) & $168.4(95.9)$ & $174.2(104.9)$ & 0.3491 \\
\hline \multicolumn{4}{|l|}{ Prescribing physician specialty, $n$ (\%) } \\
\hline Primary care & $1482(76.4)$ & $962(78.1)$ & 0.2413 \\
\hline Endocrinologist & $142(7.3)$ & $46(3.7)$ & $<0.0001$ \\
\hline Emergency medicine & $10(0.5)$ & $19(1.5)$ & 0.003 \\
\hline Other & $254(13.1)$ & $170(13.8)$ & 0.5593 \\
\hline Unknown & $53(2.7)$ & $34(2.8)$ & 0.9579 \\
\hline \multicolumn{4}{|l|}{ Index insulin, $n(\%)$} \\
\hline Insulin glargine & $1398(72.0)$ & $891(72.4)$ & 0.8277 \\
\hline Insulin detemir & $528(27.2)$ & $163(13.2)$ & $<0.0001$ \\
\hline Insulin isophane (NPH) & $15(0.8)$ & $177(14.4)$ & $<0.0001$ \\
\hline
\end{tabular}

DCSI Diabetes Complication Severity Index, Deyo-CCI Deyo-Charlson Comorbidity Index, HbAlc glycated hemoglobin, $S D$ standard deviation

${ }^{a}$ During the pre-index period

by an endocrinologist as compared with a PCP. However, all other prescriber types had a statistically significantly higher HR relative to PCP prescribers, indicating higher risk of non-persistence on basal insulin (data not shown).

\section{Change in HbA1c Values}

A subgroup of 817 patients in the pen cohort and 558 patients in the vial cohort had HbA1c laboratory values available during both the baseline and follow-up periods. The difference in $\mathrm{HbA1c}$ from baseline to follow-up was found to be significantly greater for the pen cohort than for the vial cohort $(-0.80 \%$ vs. $-0.60 \%$; $P=0.0473)$.

\section{DISCUSSION}

This study in a mostly elderly T2DM population enrolled in an MAPD plan and newly started on basal insulin therapy found significant differences in a variety of characteristics between patients using pens and those using vials and syringes. Patients using pens tended to be have fewer comorbidities, and the ethnic and geographic distributions differed between the 
Table 3 Adherence and persistence measures

\begin{tabular}{llll}
\hline Measure & Pen cohort & Vial cohort & $P$ value \\
\hline $\begin{array}{l}\text { Number of prescription claims prior to discontinuation, mean (SD) } \\
\text { Adherence }\end{array}$ & $2.93(2.9)$ & $2.13(2.5)$ & $<0.0001$ \\
Mean adherence (MPR) & $0.75( \pm 0.3)$ & $0.57( \pm 0.3)$ & $<0.0001$ \\
Mean adherence (PDC) & & & \\
Unadjusted mean (95\% CI) & $0.71(0.70,0.73)$ & $0.53(0.52,0.55)$ & $<0.0001$ \\
Adjusted mean (95\% CI) & $0.67(0.61,0.72)$ & $0.50(0.45,0.56)$ & $<0.0001$ \\
Proportion adherent (MPR $\geq 80 \%), n(\%)$ & $1123(57.9)$ & $436(35.4)$ & $<0.0001$ \\
Proportion adherent (PDC $\geq 80 \%), n(\%)$ & $959(49.4)$ & $366(29.7)$ & $<0.0001$ \\
Adjusted odds of adherence (PDC $\geq 80 \%)(95 \%$ CI) & $2.19(1.86,2.59)$ & Reference & $<0.0001$ \\
Persistence & & & $<0.0001$ \\
Unadjusted mean persistence, days (SD) & $181.0(128.1)$ & $93.7(89.8)$ & $<0.0001$ \\
Adjusted hazard ratio (95\% CI) & $0.415(0.381,0.452)$ & Reference & $<0.0001$ \\
Mean basal insulin fills prior to discontinuation & 2.93 & 2.13 &
\end{tabular}

All reported demographic and clinical characteristics were included in the adjusted models

$C I$ confidence interval, $M P R$ medication possession ratio, $P D C$ proportion of days covered, $S D$ standard deviation

two groups. However, when adjusting for the differences between the two groups, it was clear that those using pens to deliver basal insulin had a significantly higher rate of adherence and persistence than those administering basal insulin via vial and syringe. This analysis is unique in that it used the PDC, as well as the MPR, to evaluate adherence to basal insulin. Furthermore, the study population differed from those in previous studies, being an MAPD population consisting primarily of elderly health plan members (aged 65 years and older). Thus, this study provides a different perspective on insulin use among this demographic, one of the fastest growing segments of US society in general and one that has a high burden of diabetes disease.

In this analysis, the adjusted overall odds ratio favoring adherence was higher for patients using pens than vials. The adjusted mean rates of adherence ( 0.71 and 0.53 for the pen and vial cohorts, respectively) are on the high end of the ranges reported in previous studies (0.53-0.69 for the pen cohort and $0.38-0.62$ for the vial cohort) $[6,8,24]$ despite the use of the PDC calculation, which allows for a more conservative approach to evaluating medication adherence to insulin use $[6,8,11]$. This difference may be due to the use of the days' supply adjustment factor, which was $>1$ for all six of the basal insulin-delivery device combinations and, therefore, increased the days' supply of all of the pharmacy claims used in the study. These adjustments reflect the fact that most basal insulin prescriptions last longer than the 30 days' supply that is typically reported in pharmacy claims. The adjusted days' supply used in this study may more accurately represent the true number of days with insulin supply available to the 
Table 4 Logistic regression of adherence (PDC $\geq 80 \%$ )

\begin{tabular}{|c|c|c|c|c|}
\hline \multirow[t]{2}{*}{ Parameter } & \multirow[t]{2}{*}{ Odds ratio } & \multicolumn{2}{|c|}{$95 \% \mathrm{CI}$} & \multirow[t]{2}{*}{$P$ value } \\
\hline & & Lower & Upper & \\
\hline Cohort $($ pen $=1$, vial $=0)$ & 2.19 & 1.86 & 2.59 & $<0.0001$ \\
\hline Age & 1.00 & 0.99 & 1.01 & 0.638 \\
\hline Deyo-CCI score & 0.95 & 0.91 & 0.98 & 0.004 \\
\hline DCSI score & 0.98 & 0.94 & 1.02 & 0.412 \\
\hline Number of prescription claims ${ }^{a}$ & 1.00 & 1.00 & 1.00 & $<0.0001$ \\
\hline Male sex & 1.04 & 0.89 & 1.21 & 0.635 \\
\hline Count of HbAlc tests performed ${ }^{a}$ & 1.04 & 1.01 & 1.08 & 0.017 \\
\hline \multicolumn{5}{|l|}{ Index basal insulin product } \\
\hline Insulin detemir & 0.98 & 0.82 & 1.18 & 0.867 \\
\hline Insulin isophane $(\mathrm{NPH})$ & 1.26 & 0.90 & 1.75 & 0.173 \\
\hline Insulin glargine & Ref. & & & \\
\hline LIS status and dual eligibility & 1.19 & 0.89 & 1.58 & 0.244 \\
\hline Non-insulin injectable use $^{a}$ & 1.02 & 0.80 & 1.30 & 0.876 \\
\hline Count of physician office visits ${ }^{a}$ & 1.00 & 0.99 & 1.00 & 0.009 \\
\hline Oral antidiabetic use ${ }^{a}$ & 1.21 & 0.93 & 1.57 & 0.162 \\
\hline \multicolumn{5}{|l|}{ Index prescriber type } \\
\hline Endocrinologist & 0.96 & 0.71 & 1.32 & 0.819 \\
\hline ER physician & 0.40 & 0.16 & 1.02 & 0.055 \\
\hline Other & 0.72 & 0.58 & 0.90 & 0.004 \\
\hline Unknown & 0.70 & 0.44 & 1.12 & 0.136 \\
\hline Primary care & Ref. & & & \\
\hline \multicolumn{5}{|l|}{ Population density } \\
\hline Suburban & 1.23 & 1.03 & 1.47 & 0.020 \\
\hline Rural & 1.33 & 1.04 & 1.70 & 0.025 \\
\hline Unknown & 0.78 & 0.31 & 1.98 & 0.603 \\
\hline Urban & Ref. & & & \\
\hline \multicolumn{5}{|l|}{ Geographic region } \\
\hline Northeast & 1.52 & 0.87 & 2.66 & 0.144 \\
\hline Midwest & 1.47 & 1.23 & 1.76 & $<0.0001$ \\
\hline West & 1.56 & 1.20 & 2.02 & 0.001 \\
\hline
\end{tabular}


Table 4 continued

\begin{tabular}{|c|c|c|c|c|}
\hline \multirow[t]{2}{*}{ Parameter } & \multirow[t]{2}{*}{ Odds ratio } & \multicolumn{2}{|c|}{$95 \% \mathrm{CI}$} & \multirow[t]{2}{*}{$P$ value } \\
\hline & & Lower & $\overline{\text { Upper }}$ & \\
\hline South & \multicolumn{4}{|l|}{ Ref. } \\
\hline \multicolumn{5}{|c|}{$\begin{array}{l}\text { Dependent variable for logistic regression model }=\text { adherent. Odds ratios }>1.0 \text { indicate higher odds of being adherent } \\
C C I \text { Charlson Comorbidity Index score, DCSI Diabetes Complication Severity Index score, ER emergency room, LIS low } \\
\text { income subsidy, PDC proportion of days covered, ref. reference } \\
\text { a During the pre-index period }\end{array}$} \\
\hline
\end{tabular}

patients, thus providing a more accurate calculation of adherence and persistence to insulin therapy. The difference in adherence may also be a result of the differences between the populations that could not be accounted for using the available data, and the use of PDC for calculating adherence as opposed to the MPR calculation used in previous investigations $[6,8$, 11].

The regression analyses examining characteristics associated with adherence and persistence to basal insulin therapy showed several similarities. First, mean adherence and persistence were higher in the pen cohort, which corroborates findings from previous studies $[6,8,11]$. Second, patients' geographic region and population density were observed to affect adherence and persistence. Residence outside of the Southern US showed positive associations with both adherence and persistence. The reasons for these associations are not immediately evident, but are undoubtedly multifactorial.

Among the subset of patients for whom preand post-index HbA1c data were available, larger decreases in $\mathrm{HbA1c}$, indicating better glycemic control, were observed in the pen cohort compared with the vial cohort. While not a primary outcome of this study, this subgroup analysis showed a trend between higher adherence and larger decreases in
HbA1c values. Long-term investigations of the effect of glycemic control on outcomes have demonstrated that improvements in glycemic control are associated with decreases in both microvascular and neuropathic complications of diabetes [25-27] and have shown trends suggesting lower overall cardiovascular risks [28].

Interestingly, the baseline characteristics of the two cohorts highlighted that pen use was more prevalent in suburban areas and less prevalent in urban areas. Patients in urban areas were more likely to use vials over pen devices. Furthermore, a higher proportion of the pen cohort resided in the Midwest, while a higher proportion of the vial cohort resided in the South. This suggests that there may be regional differences in providers' prescribing patterns. Additional examination of this trend is needed to understand the implications of these observations.

There have been several previous studies comparing rates of adherence [6-8, 11, 24, 29] and persistence $[6,8,12]$ among patients using insulin pens versus vials using administrative claims databases. The results of the current study support the findings from previous studies that showed higher rates of adherence with insulin pens than with vials and syringes. For example, Buysman et al. [6] compared adherence and persistence between patients 
initiating basal insulin therapy with either insulin detemir in a pen or insulin isophane (NPH) in a vial, and found that patients on the insulin detemir pen had 39\% higher odds of adherence (MPR $\geq 80 \%$ ) and a 38\% lower hazard of discontinuation. A similar investigation by Davis et al. [12] showed that patients initiating insulin glargine in a pen versus vial were significantly less likely to discontinue therapy or switch to another therapy. In several studies that also reported costs, the improved adherence and/or persistence was achieved without increasing overall healthcare costs [7, 11-13, 24]. Miao et al. [13], in a study based on a Medicare population, noted better persistence and adherence with insulin therapy prescribed as a pen compared with a vial, with no increase in total health care costs.

Several studies have examined the impact of adherence to oral antidiabetic therapies on clinical and economic outcomes [20-37]. These other investigations have included outcome measures such as glycemic control (HbA1c laboratory values) $[35,36]$, health care resource utilization [31, 32, 34, 37], and healthcare costs [33, 37], all of which were found to improve with higher rates of adherence, and are in agreement with results in the current study with insulin therapy.

The study cohorts were found to be significantly different in several important baseline demographic and clinical characteristics, which partly could have been due to the large numbers of patients in each cohort. Multivariate analyses were used to adjust for many of the characteristics known to be associated with medication adherence and persistence, such as age and level of comorbidity. However, there may be additional confounders that were not available for inclusion.
While the PDC, which is a more conservative measure of adherence, was chosen in the present study, this method does have limitations. For example, in calculating of PDC, it was not possible to adjust the starting date for overlapping prescription fills. Therefore, this method does not account for patients who may fill a prescription early but continue to use the remaining supply from the earlier prescription and may, therefore, underreport adherence.

There are other limitations inherent to claims database research. First, claims data are prone to coding errors, and they may not reflect actual disease or therapies. While pharmacy claims accurately represent all prescriptions that are filled, they may not give a true depiction of actual medication use. It was assumed that patients were filling insulin prescriptions as the drug was needed, and they were not hoarding, wasting, or discarding unused insulin. Furthermore, pharmacy claims are only available for prescriptions that were paid through the patients' insurance. Administrative datasets do not capture instances where patients received drug samples or paid cash for their prescription. However, this was a cohort comparison study and there is no reason to expect that the rate of any of these occurrences would have differed between the two study groups. In addition, a days' supply within pharmacy claims often does not provide a true representation of the days that could potentially be covered given each patient's dosage, as demonstrated by the median days' supply of 30 days for all six basal insulin-delivery device combinations in this study. The adjustment factor used in the present study was an approximation meant to account for the general pattern of insulin fills and provides an estimated days' supply that 
the authors believe is more accurate than the days' supply directly from pharmacy claims. The study population was comprised of MAPD members from a large national managed care organization. However, these patients were residing primarily in the southern and Midwestern states of the US. Therefore, the results of the study may not be generalizable beyond the studied health plan. Additionally, because this was an observational, retrospective claims-based study, its results are indicative of associations of time with medication use variables rather than any causal relationships among those variables. Nonetheless, the results of this study draw attention to important relationships that govern medication adherence and persistence and may warrant further exploration.

\section{CONCLUSIONS}

Elderly patients with T2DM often suffer from multiple comorbid conditions and may find it difficult to maintain adherence and persistence to insulin therapy, which compromise glycemic control. This study in a mostly elderly MAPD population found that patients using pens and those using vials and syringes to administer basal insulin had some differing characteristics, but following adjustments for these differences, a significantly higher rate of adherence and persistence was noted with use of a pen device for insulin delivery than when using vials and syringes. While it seems intuitive that pens would offer a greater degree of convenience and simplicity than vials and syringes, additional research is needed to further examine the clinical outcomes and economic ramifications of these administration techniques in an elderly demographic.

\section{ACKNOWLEDGMENTS}

This study was supported by a research partnership between Novo Nordisk, Inc. (Plainsboro, NJ, USA) and Humana, Inc. (Louisville, KY, USA). Funding for manuscript preparation and submission was provided by Novo Nordisk, Inc. The article processing charges and open access fee for this publication were funded by Novo Nordisk, Inc. All named authors meet the International Committee of Medical Journal Editors (ICMJE) criteria for authorship for this manuscript, take responsibility for the integrity of the work as a whole, and have given final approval to the version to be published. Mary Costantino, $\mathrm{PhD}$ (Comprehensive Health Insights, Inc., Louisville, KY, USA) and Tulay Cushman (Novo Nordisk, Inc.) contributed to manuscript preparation. Editorial assistance was provided by Ed Kimball, PhD (Novo Nordisk, Inc.) and Anthony Shardt, MD (Churchill Communications, Maplewood, NJ, USA). Support for all writing and editing assistance was provided by Novo Nordisk, Inc.

Disclosures. SLS, YL, and DCM were all employees of Comprehensive Health Insights, Inc. at the time this research was conducted. SLS is currently an employee and stock-holder of Humana, Inc. YAM is an employee of Humana, Inc. and provided input on the study design, interpretation of the results, and manuscript. JCB was an employee of Humana, Inc. at the time that this research was conducted and provided input on the study design, interpretation of the results, and manuscript. JRB is an employee and stock-holder of Novo Nordisk, Inc. and provided input on the study design, interpretation of the results, and preparation of the manuscript. 
Compliance with ethics guidelines. All procedures followed were in accordance with the ethical standards of the responsible committee on human experimentation (institutional and national) and with the Helsinki Declaration of 1964, as revised in 2013. This study was based on claims data and did not include any identifying patient information. The research protocol was reviewed and approved prior to study initiation by an independent Institutional Review Board and was granted a waiver of Health Insurance Portability and Accountability Act authorization and a waiver of informed consent.

Open Access. This article is distributed under the terms of the Creative Commons Attribution-NonCommercial 4.0 International License (http://creativecommons.org/licenses/ by-nc/4.0/), which permits any noncommercial use, distribution, and reproduction in any medium, provided you give appropriate credit to the original author(s) and the source, provide a link to the Creative Commons license, and indicate if changes were made.

\section{REFERENCES}

1. Garber AJ, Abrahamson MJ, Barzilay JI, et al. AACE comprehensive diabetes management algorithm 2013. Endocrin Pract. 2013;19(2):327-36.

2. Inzucchi SE, Bergenstal RM, Buse JB, et al. Management of hyperglycemia in type 2 diabetes: a patient-centered approach: position statement of the American Diabetes Association (ADA) and the European Association for the Study of Diabetes (EASD). Diabetes Care. 2012;35(6):1364-79.

3. American Diabetes Association. Standards of medical care in diabetes-2014. Diabetes Care. 2014;37(Suppl 1):S14-S80.

4. Owens DR. Clinical evidence for the earlier initiation of insulin therapy in type 2 diabetes. Diabetes Technol Ther. 2013;15(9):776-85.
5. Meece J. Dispelling myths and removing barriers about insulin in type 2 diabetes. Diabetes Educ. 2006;32(1 Suppl):9S-18S.

6. Buysman E, Conner C, Aagren M, Bouchard J, Liu F. Adherence and persistence to a regimen of basal insulin in a pre-filled pen compared to vial/syringe in insulin-naïve patients with type 2 diabetes. Curr Med Res Opin. 2011;27(9):1709-17.

7. Grabner M, Chu J, Raparla S, Quimbo R, Zhou S, Conoshenti J. Clinical and economic outcomes among patients with diabetes mellitus initiating insulin glargine pen versus vial. Postgrad Med. 2013;125(3):204-13.

8. Lee WC, Balu S, Cobden D, Joshi AV, Pashos CL. Medication adherence and the associated health-economic impact among patients with type 2 diabetes mellitus converting to insulin pen therapy: an analysis of third-party managed care claims data. Clin Ther. 2006;28(10):1712-25.

9. Wright BM, Bellone JM, McCoy EK. A review of insulin pen devices and use in the elderly diabetic population. Clin Med Insights Endocrinol Diabetes. 2010;3:53-63.

10. Asche CV, Luo W, Aagren M. Differences in rates of hypoglycemia and health care costs in patients treated with insulin aspart in pens versus vials. Curr Med Res Opin. 2013;29(10):1287-96.

11. Baser O, Bouchard J, DeLuzio T, Henk H, Aagren M. Assessment of adherence and healthcare costs of insulin device (FlexPen) versus conventional vial/ syringe. Adv Ther. 2010;27(2):94-104.

12. Davis SN, Wei W, Garg S. Clinical impact of initiating insulin glargine therapy with disposable pen versus vial in patients with type 2 diabetes mellitus in a managed care setting. Endocr Pract. 2011;17(6):845-52.

13. Miao R, et al. Does device make any difference? A real-world retrospective study of insulin treatment among elderly patients with type 2 diabetes. J Diabetes Sci Technol. 2014;8(1):150-8.

14. Bethel MA, Sloan FA, Belsky D, Feinglos MN. Longitudinal incidence and prevalence of adverse outcomes of diabetes mellitus in elderly patients. Arch Intern Med. 2007;167(9):921-7.

15. Ligthelm RJ, Kaiser M, Vora J, Yale J-F. Insulin use in elderly adults: risk of hypoglycemia and strategies for care. J Am Geriatr Soc. 2012;60(8):1564-70.

16. Sue Kirkman M, Briscoe VJ, Clark N, et al. Diabetes in older adults: a consensus report. J Am Geriatr Soc. 2012;60(12):2342-56. 
17. Hart LG, Larson EH, Lishner DM. Rural definitions for health policy and research. Am J Public Health. 2005;95(7):1149-55.

18. West AN, Weeks WB, Wallace AE. Rural veterans and access to high-quality care for high-risk surgeries. Health Serv Res. 2008;43(5 Pt 1):1737-51.

19. Deyo RA, Cherkin DC, Ciol MA. Adapting a clinical comorbidity index for use with ICD-9-CM administrative databases. J Clin Epidemiol. 1992;45(6):613-9.

20. Young BA, Lin E, Von Korff M, et al. Diabetes complications severity index and risk of mortality, hospitalization, and healthcare utilization. Am J Manag Care. 2008;14(1):15-23.

21. Chang H-Y, Weiner JP, Richards TM, Bleich SN, Segal JB. Validating the adapted Diabetes Complications Severity Index in claims data. Am J Manag Care. 2012;18(11):721-6.

22. Martin BC, Wiley-Exley EK, Richards S, et al. Contrasting measures of adherence with simple drug use, medication switching, and therapeutic duplication. Ann Pharmacother. 2009;43:36-44.

23. Nau DP. Proportion of days covered (PDC) as a preferred method of measuring medication adherence. http://www.pqaalliance.org/images/ uploads/files/PQA\%20PDC\%20vs\%20\%20MPR.pdf. Accessed May 14, 2015.

24. Lee LJ, Li Q, Reynolds MW, Pawaskar MD, Corrigan SM. Comparison of utilization, cost, adherence, and hypoglycemia in patients with type 2 diabetes initiating rapid-acting insulin analog with prefilled pen versus vial/syringe. J Med Econ. 2011;14(1):75-86.

25. The effect of intensive treatment of diabetes on the development and progression of long-term complications in insulin-dependent diabetes mellitus. The Diabetes Control and Complications Trial Research Group. N Engl J Med. 1993;329(14):977-86.

26. Effect of intensive blood-glucose control with metformin on complications in overweight patients with type 2 diabetes (UKPDS 34). UK Prospective Diabetes Study (UKPDS) Group. Lancet. 1998;352(9131):854-65.

27. Ohkubo Y, Kishikawa H, Araki E, et al. Intensive insulin therapy prevents the progression of diabetic microvascular complications in Japanese patients with non-insulin-dependent diabetes mellitus: a randomized prospective 6-year study. Diabetes Res Clin Prac. 1995;28(2):103-17.

28. Nathan DM, Cleary PA, Backlund J-YC, et al. Intensive diabetes treatment and cardiovascular disease in patients with type 1 diabetes. N Engl J Med. 2005;353(25):2643-53.

29. Asche CV, Shane-McWhorter L, Raparla S. Health economics and compliance of vials/syringes versus pen devices: a review of the evidence. Diabetes Technol Ther. 2010;12(Suppl 1):S101-8.

30. Cramer JA. A systematic review of adherence with medications for diabetes. Diabetes Care. 2004;27(5):1218-24.

31. Hepke KL, Martus MT, Share DA. Costs and utilization associated with pharmaceutical adherence in a diabetic population. Am J Manag Care. 2004;10(2 Pt 2):144-51.

32. Ho PM, Rumsfeld JS, Masoudi FA, et al. Effect of medication nonadherence on hospitalization and mortality among patients with diabetes mellitus. Arch Intern Med. 2006;166(17):1836-41.

33. Hong JS, Kang HC. Relationship between oral antihyperglycemic medication adherence and hospitalization, mortality, and healthcare costs in adult ambulatory care patients with type 2 diabetes in South Korea. Med Care. 2011;49(4):378-84.

34. Lau DT, Nau DP. Oral antihyperglycemic medication nonadherence and subsequent hospitalization among individuals with type 2 diabetes. Diabetes Care. 2004;27(9):2149-53.

35. Pladevall M, Williams LK, Potts LA, Divine G, Xi H, Lafata JE. Clinical outcomes and adherence to medications measured by claims data in patients with diabetes. Diabetes Care. 2004;27(12):2800-5.

36. Rozenfeld Y, Hunt JS, Plauschinat C, Wong KS. Oral antidiabetic medication adherence and glycemic control in managed care. Am J Manag Care. 2008;14(2):71-5.

37. Sokol MC, McGuigan KA, Verbrugge RR, Epstein RS. Impact of medication adherence on hospitalization risk and healthcare cost. Med Care. 2005;43(6):521-30. 